13. Josefsson PO, Johnell O, Wendeberg B (1987) Ligamentous injuries in dislocations of the elbow joint. Clin Orthop Relat Res 221:221e5

14. Lill H, Korner J, Rose T et al (2001) Fracture - dislocations of the elbow joint - strategy for treatment and results. Arch Orthop Trauma Surg 121:31-37

15. Markgraf E, Mohr S, Kolb W (2002) Ellenbogenluxation - Bewertung, Begleitschäden, Therapie. Trauma Berufskrankh 4(Suppl 1):565-567

16. Michels F, Pouliart N, Handelberg F (2007) Arthroscopic management of Mason type 2 radial head fractures. Knee Surg Sports Traumatol Arthrosc 15(10):1244-1250

17. Mitani M, Nabeshima Y, Ozaki A et al (2009) Arthroscopic reduction and percutaneous cannulated screw fixation of a capitellar fracture of the humerus: a case report. J Shoulder Elbow Surg 18(2):e6e9

18. Morrey BF(1995) Current concepts in treatment of fractures of the radial head, the olecranon and the coronoid. J Bone Joint Surg 77:316-327

19. Perez Carro L, Golano P, Vega J (2007) Arthroscopic-assisted reduction and percutaneous external fixation of lateral condyle fractures of the humerus. Arthroscopy 23(10):1131.e1-1131.e4

20. Riel KA, Bernett P (1993) Die einfache Ellenbogenluxation. Vergleich der Langzeitergebnisse nach Ruhigstellung und funktioneller Behandlung. Unfallchirurg 96:529-533

21. Robinson CM, Hill RM, Jacobs $\mathrm{N}$ et al (2003) Adult distal humeral metaphyseal fractures: epidemiology results of treatment. J Orthop Trauma 17:38-47

22. Rolla PR, Surace MF, Bini A, Pilato G (2006) Arthroscopic treatment of fractures of the radial head. Arthroscopy 22(2):233.e1-233.e6

23. Rommens PM, Küchle R, Schneider RU, Reuter M (2004) Olecranonfractures in adults: factors influence in outcome. Injury 35:1149-1157

24. Rueger JM, Jannssen A, Barvencik F, Briem D (2005) Distale Humerusfraktur. Unfallchirurg 108:49-58

25. Srinivasav K, Agarwal M, Matthews SJE, Giannoudis PV (2005) Fractures of the distal humerus in elderly. Clin Orthop Relat Res 434:220-230

26. Van Tongel A, Macdonald P, Van Riet R, Dubberley $J$ (2012) Elbow arthroscopy in acute injuries. Knee Surg Sports Traumatol Arthrosc 20(12):2542-2548

27. Wang J, Liu Y, Li Z et al (2012) Arthroscopic poking reduction and internal fixation of radial head fractures. Zhongguo Xiu Fu Chong Jian Wai Ke Za Zhi 26(2):208-211

28. Wanner $G$, Rechel $H$, Frank J et al (2006) Halte- und Bewegungsapparat. In: Bruch HP, Trentz O (Hrsg) Berchthold Chirurgie, 5. Aufl. Elsevier GmbH, München, $S$ 330-544

29. Werner C, Trentz O (2003) Ellenbogengelenk. In Rüter A, Trentz O, Wagner M (Hrsg) Unfallchirurgie. Urban \& Fischer, S 801-816

30. Wijeratna M, Bailey KA, Pace A et al (2012) Arthroscopic radial head excision in managing elbow trauma. Int Orthop 36(12):2507-2512

Arthroskopie 2014 · 27:282 DOI 10.1007/s00142-014-0843-7

Online publiziert: 7. November 2014

(c) Springer-Verlag Berlin Heidelberg 2014

\title{
C. Krauss
}

Abteilung für Sportorthopädie, Orthopädische Fachklinik, Marienkrankenhaus, Düsseldorf-Kaiserswerth

\section{Erratum zu: Schmerzhafte und kraftlose Schulter ohne aktuelle Traumaanamnese}

Leider enthält der Beitrag einen inhaltlichen Fehler. In Bezug auf Abb.1 lautet die korrekte Bildlegende: MRT-Untersuchung in STIR-Sequenz koronar und transversal: Bei jeweils intakter Sehne zeigt sich eine Signalerhöhung im Bereich des $M$. supraspinatus und $M$. infraspinatus, welche als Ödem bei neuronaler Störung zu interpretieren ist. Die nichtabgebildete koronare T1-Aufnahme ist unauffällig.

In Bezug auf Abb. 2 lautet die korrekte Aussage im Fließtext: Auffällig ist eine deutliche Atrophie des M. supraspinatus (SSP) und des M. infraspinatus (ISP) entsprechend einem Stadium II nach Goutallier mit positivem Tangentenzeichen.

Analog dazu lautet die korrekte Bildlegende zu Abb.2: T2-gewichtete Sequenz nicht fettsupprimert sagittal: deutliche Atrophie und diffuse Signal- erhöhung im M. supraspinatus sowie M. infraspinatus.

Wir bitten den Fehler zu entschuldigen und den korrekten Wortlaut zu beachten.

\section{Korrespondenzadresse}

\section{Dr. C. Krauss}

Abteilung für Sportorthopädie, Orthopädische Fachklinik, Marienkrankenhaus

An St. Swidbert 17,

40489 Düsseldorf-Kaiserswerth

christine.krauss@vkkd-kliniken.de

Die Online-Version des Originalartikels können Sie unter http://dx.doi.org/ 10.1007/s00142-014-0808-x finden. 